\title{
New approach for layer thickness measurements using pulsed lock-in thermography
}

\author{
by D. Hoffmann ${ }^{\star}$, T. Hochrein ${ }^{\star}$, M. Bastian*, G. Schober ${ }^{\star}$ \\ "SKZ-German Plastics Center, Friedrich-Bergius-Ring 22, 97076 Wuerzburg, Germany, d.hoffmann@skz.de
}

\begin{abstract}
The coating of materials has grown to an important treatment for improving functionality and appearance of components. Active thermography has been established as a method for quality monitoring, but the required excitation sources for lock-in thermography are either very expensive or too inert. Pulse thermography is more cost-effective but results in a lower signal-to-noise-ratio (SNR). Here, a novel approach is presented where the sample is periodically excited with consecutive high energy pulses and submitted to a lock-in evaluation. This so-called pulsed lock-in thermography offers a less expensive and reproducible way for nondestructive testing and has the potential for measuring the thickness of coatings.
\end{abstract}

\section{State of the Art}

Active thermography is a non-destructive and contact-free technique for the inspection and characterization of components. In the case of lock-in thermography the sample is thermally excited with an amplitude or a frequency modulated signal from an external source. The excitation is most commonly induced optically, e.g. by halogen lamps, by an LED array or by lasers [1,2,3]. The thinner the layer to be characterized, the higher is the needed modulation frequency. For the investigation of coatings frequencies in the range of several Hertz are needed $[4,5]$. However, existing optical excitation sources are either too inert (e.g. halogen lamps) or very expensive (e.g. laser). In contrast, using pulse thermography the test object is heated with a short and intense thermal pulse, e.g. by means of powerful flashbulbs, an LED array or lasers as well $[2,6,7]$. Due to the short excitation time and high responsiveness of the excitation source high frequencies can be accomplished, which allows the investigation of thin layers. However, the corresponding amplitude and phase values calculated for each frequency are accompanied by a lower SNR compared to the results from lock-in measurements.

In both cases the excitation leads to a heat wave propagation from the surface into the interior of the test object. Interfaces between coating and substrate influence this behavior and thus lead to variations in the temperature distribution on the surface. Temporal variations in the appearance depend on the depth of the interface and thus the thickness of the layer [1]. By using thermography cameras, the temperature behavior of the surface can be monitored. Depending on the evaluation method, the thermographic result is an image with a pixel-wise plotted temperature, amplitude or phase value, respectively.

\section{Pulsed lock-in thermography}

Pulsed lock-in thermography (PLT) is a special case of pulse phase thermography (PPT) [8], where the sample is excited with a sequence of consecutive flashes instead of one single flash. For characterizing coatings frequencies in the range of several hertz are needed [4], which can be accomplished due to the high responsiveness of flash lamps. In contrast to conventional pulse thermography, the multi-pulse excitation leads to a higher penetration depth and an enhanced SNR. The modulation frequency for lock-in evaluation is given by the temporal distance between the pulses. By a fast Fourier transform (FFT) analysis at the respective frequency, it is possible to obtain low-noise phase images where the phase angle correlates with the coating thickness. Thus the advantages of conventional lock-in (high SNR) and pulse thermography (high frequencies) are combined in this novel technique while the disadvantages of both methods (e.g. expensive excitation sources) are avoided.

\section{Experiment and results}

The applicability of the novel technique for thickness measurements was tested on an aluminum plate that was coated with acrylic paint. Acrylic paint represents one of the most commonly used coatings, which is applied in the craft sector, for instance. The number of layers is modulated over the sample leading to a step-like coating with thickness from $40 \mu \mathrm{m}$ to $210 \mu \mathrm{m}$. Reference measurements were taken with a micrometer on three positions at each layer. The setup of pulsed lock-in thermography consists of a flash unit with integrated pulse generator (Hensel-Visit GmbH \& Co. KG, Wuerzburg) for pulse excitation, the infrared camera thermolMAGER TIM 450 (Micro-Epsilon Messtechnik GmbH \& Co. $\mathrm{KG}$, Ortenburg) for data acquisition and a vise fixing the sample in front of lamp and camera. For the measurement a pulse trigger frequency of $1 \mathrm{~Hz}$, a pulse energy of $500 \mathrm{~J}$ and a data acquisition frequency of $27 \mathrm{~Hz}$ were used. The sample was excited with thirty pulses, as seen in the temperature-time diagram in figure 1 (left), which shows the temperature behavior of a corresponding measuring point placed in the middle part of the sample. In the upper right 
picture the resulting phase image after FFT can be seen, while the values along the dashed line in the image are illustrated in the lower right diagram together with the reference thickness values. The oscillating course of the temperature peaks visible in the temperature-time diagram can be led back to the discreet resolution of the thermal impulses depending on the acquisition frequency. This is a metrological problem and does not influence the heating behavior as seen in the constant temperature increase. The phase image reveals the potential of pulsed lock-in thermography to resolve coating thickness in the $\mu \mathrm{m}$ range as the individual layers can be differentiated from each other. The step-like increase in the thickness is visible in the line course. Deviations from an ideal echelon form can be ascribed to irregularities in the thickness coming from the manual coating process. The correlation between phase and thickness values and hence the creation of a calibration curve is currently being investigated.
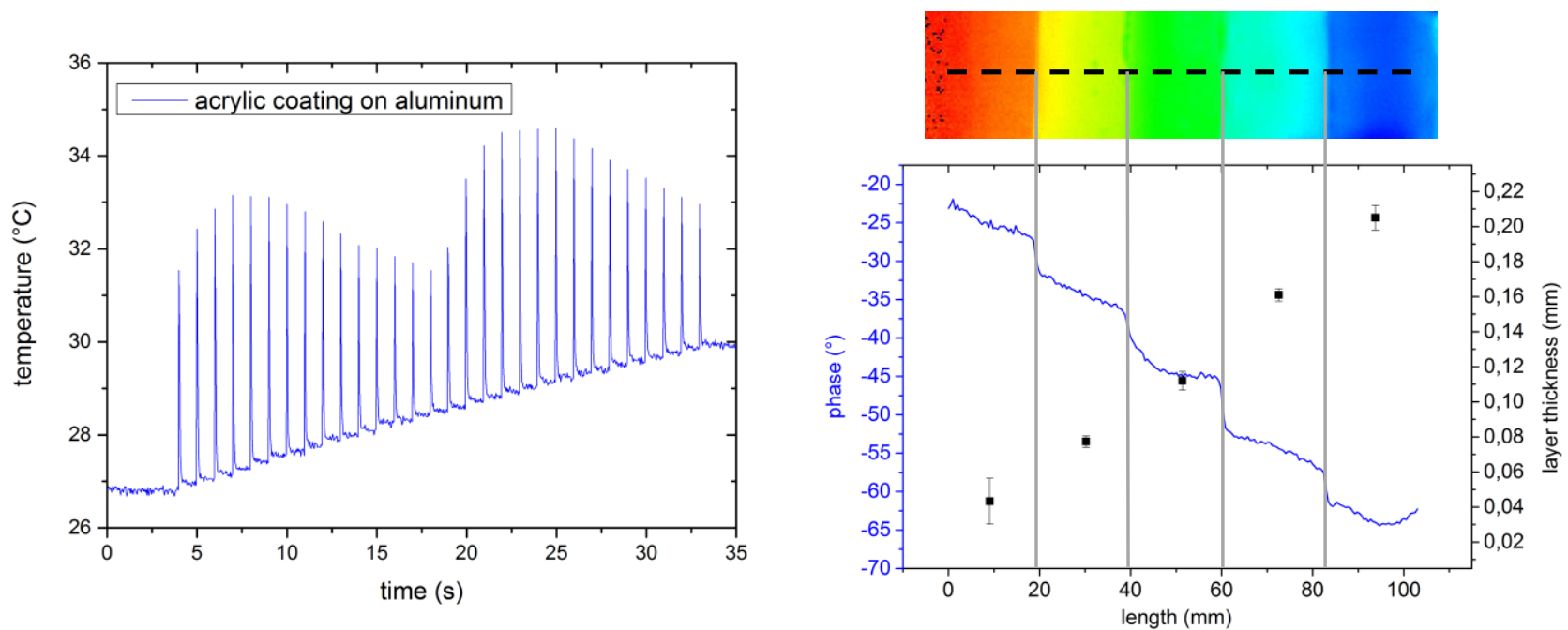

Fig. 1: Thermal response of the test object after applying a pulse sequence of thirty pulses with a temporal distance of $1 \mathrm{~s}$. The temperature was measured by the TIM 450 with an acquisition frequency of $27 \mathrm{~Hz}$ (left). Phase image of the coated aluminum plate with different layer thicknesses (right, top) as well as line course of the phase values along the dashed line in the phase image (right, bottom). The thickness values of each layer were measured with a micrometer at three positions, respectively.

\section{Conclusion}

With the presented thermography technique layers with varying thickness on a $\mu \mathrm{m}$-scale can be distinguished. Therefore pulsed lock-in thermography offers an inexpensive and reproducible way for large-area monitoring of coating layers (e.g. paint or varnish) with a relative high SNR. Additionally, due to the short measurement time the potential for inline applications is given.

\section{REFERENCES}

[1] Busse G, Wu D, Karpen W. Thermal wave imaging with phase sensitive modulated thermography. In: Journal of Applied Physics, 71.8, 1992: 3962-3965.

[2] Pickering SG, Chatterjee K, Almond DP, et al. LED optical excitation for the long pulse and lock-in thermographic techniques. In: NDT\&E International, 58, 2013: 72-77.

[3] Ziegler M, Thiel E, Ahmadi S. Lock-in thermography using high-power laser sources. In: Proceedings of ECNDT, 2018.

[4] Lindemann S, Schaller R. Laser-angeregte Lockin-Thermografie zur zerstoerungsfreien und beruehrungslosen Schichtdickenmessung von thermisch gespritzten Beschichtungen. Proceedings of DGZfP-Jahrestagung 2017.

[5] Zhang JY, Meng XB, Ma YC. A new measurement method of coatings thickness based on lock-in thermography. In: Infrared Physics \& Technology, 76, 2016: 655-660.

[6] Just P, Rost J, Echelmeyer T, et al. A method to quantify coating thickness and porosity of electrodes for lithium-ion-batteries. In: Measurement, 89, 2016: 312-315.

[7] Holland SD, Reusser RS. Material evaluation by infrared thermography. Annual Review of Materials Research, 46, 2016: 287-303.

[8] Maldague X, Marinetti S. Pulse phase infrared thermography. In: Journal of Applied Physics, 79.5, 1996: 2694-2698. 